Research Article

\title{
Vegetation Classification and Habitat Types of Gambella National Park
}

\author{
Gatluak Rolkier (iD and Kumelachew Yeshitela \\ Ethiopian Institute of Architecture, Building Construction and City Development, Addis Ababa University, P.O. Box 518, \\ Addis Ababa, Ethiopia \\ Correspondence should be addressed to Gatluak Rolkier; grolkier@gmail.com
}

Received 30 September 2019; Accepted 30 March 2020; Published 16 June 2020

Academic Editor: Anna Źróbek-Sokolnik

Copyright (c) 2020 Gatluak Rolkier and Kumelachew Yeshitela. This is an open access article distributed under the Creative Commons Attribution License, which permits unrestricted use, distribution, and reproduction in any medium, provided the original work is properly cited.

\begin{abstract}
Gambella National Park has a diverse set of habitat types which Ethiopia shares with its neighbor, South Sudan, and the park is considered as one of the top wildlife areas of Ethiopia. The objectives of this research were to determine vegetation types and identify habitat types on recent satellite imageries. The method used for vegetation data collection was transects lines. PC-ORD software was used for analyzed vegetation data while Rapid Eye image $5 \mathrm{~m}$ resolution 2012 was analyzed by ArcGIS version 10.1 to classify the habitats map of Gambella National Park. The cluster analysis classified the Gambella National Park into 6 vegetation communities, and the relative abundance and relative frequency were used for naming vegetation community types. However, the satellite image had classified the Gambella National Park into 5 major habitat types.
\end{abstract}

\section{Introduction}

Gambella National Park was established in 1973 and has a diverse set of habitat types which Ethiopia shares with its neighbor, South Sudan [1]. Vast collections of plains game are found in the park and perhaps that can be considered as one of the top wildlife areas of Ethiopia [1]. The major vegetation types that are observed in the park are woodland, wooded grassland, grassland, and wetlands. Since the 1980s, there has been large scale habitats changed in Gambella National Park mostly due to human pressure. These pressures came from establishment of state farm in Abobo with the area of 3,000 ha of land, situated in the eastern part of Gambella National Park and the Construction of Alewero dam for large scale commercial agriculture [2]. At present, both large scale agricultural investments (e.g., like Karaturi, Rushi, and Saudi Star) [3] and small scale agricultural investments from different national investors reduced the park area from $5,061 \mathrm{~km}^{2}$ to $4,575 \mathrm{~km}^{2}$. It is assumed that these anthropogenic impacts have affected wildlife and their habitats.
The development of management plan of the Gambella National Park is hindered by lack of information on habitat types within the park. Although there have been few studies focusing on the vegetation of Gambella region as a whole $[4,5]$, identification and mapping of the habitat types and distribution of wildlife within the national park have never been attempted. This is particularly important for planning sustainable wildlife management. Mapping of wildlife habitat could be used as a tool in wildlife management, a guide for wildlife viewing, and a gauge for the loss of critical wildlife habitats [6].

The main reason for classifying and mapping the habitats of Gambella National Park was to have better understanding on the location of vegetation community types at local level and habitats types at large level. This could allow better understanding of the important habitat types for key conservation species in which the park was established for. This research expected to fulfill a knowledge gap on the information of vegetation communities and habitat types for Gambella National Park.

The objectives of this research are as follows: 
To determine the vegetation types in the Gambella National Park

To identify the habitat types on the recent satellite imageries and develop a habitat map of Gambella National Park

\section{Materials and Methods}

Gambella National Park is located in the lowland plain of the Gambella People's National Regional State of Ethiopia. According to Monico et al. [7], the park is situated between $32^{\circ} 59^{\prime}$ and $35^{\circ} 23^{\prime}$ longitude and $6^{\circ} 17^{\prime}$ and $8^{\circ} 42^{\prime}$ latitude (Figure 1). It is situated between two major rivers Baro and Akobo (Figure 1) and crossed by other three major rivers with three wetlands (Figure 1). It was established in 1973, with new area of $4,575 \mathrm{~km}^{2}$.

\section{Methods of Data Collection and Analysis}

\subsection{Data Collection}

3.1.1. Vegetation Data Sampling. The method employed for vegetation data collection was systematic sampling. This sampling was done online transects which were laid down across east to west and north to south depending on habitat information (anthropogenic disturbance, physiognomy, etc.), which was designed based on identified areas with high environmental variability. This technique was used because it is simple for ecological surveys and good for sampling a very large area relatively quickly [8].

3.1.2. Data Collection. The vegetation data were collected by transects lines. The first transect was established based on vegetation physiognomy and by avoiding sites of severe human impact, e.g., fire. The remaining transects were put in place systematically at specific intervals of $5 \mathrm{~km}$. In the woodland and wooded grassland areas, sample plots of $40 \mathrm{~m} \times 40 \mathrm{~m}$ were laid out at intervals of $500 \mathrm{~m}$ along the transect for recording tree and shrub species. For recording herbaceous and grass species, four, $2 \mathrm{~m} \times 2 \mathrm{~m}$ sample plots were laid out at the corners of the bigger plot in grassland, savannah, and wetland areas; sample plots of $2 \mathrm{~m} \times 2 \mathrm{~m}$ were laid at intervals of $500 \mathrm{~m}$ apart for recording herbaceous and grass species. Accordingly, a total of 450 sample plots, woodland (292 plots), wooded grassland (98 plots), grassland (35 plots), savanna (11 plots), and wetland (14 plots), were sampled. Due to inconvenience for these much sample plots for analysis, the screening was done based on species area curve and similarity of the species and, therefore, sample plots with most similar species were excluded from the analysis.

Consequently, 80 sample plots, woodland (26 plots), wooded grassland (20 plots), grassland (12 plots), savanna (8 plots), and wetland (14 plots), were taken for final analysis.

In each sample plot the GPS point, altitude and species list, which included the habits, were recorded. Every plant species was recorded with its estimated percent cover abundance using the scale of Braun Blanquette 1932 as modified by Westhoff and Vander Maarel [9], with 1-2 individuals covering $<5 \%$ of the sampled area, 3-10 individuals covering $<5 \%$ of sampled area, abundance individuals covering $<5 \%$ of the sampled area, plant cover ranging from 5 to $12 \%$, plant covering from 12 to $25 \%$, plant covering from 25 to $50 \%$, plant covering from 50 to $75 \%$, and plant covering from 75 to $100 \%$.

Common plant species were identified in the field and for unidentified 10 species herbarium specimens were taken and identified in the National Herbarium of Ethiopia by comparing them with already identified plant species and referring to the "Flora of Ethiopia and Eritrea" (Vol. 1, Hederg et al. [10]; Vol: 2.1 Edwards et al. [11]; Vol: 2.2 Edwards et al. [12]; Vol: 3 Hederg et al. [13]; Vol 4: 1 Hederg et al. [14]; Vol: 5 Hederg et al. [15, 16]; Vol: 7 Hederg et al. [17]; and Vol: 8 Hederg et al. [10]).

\subsection{Data Analysis}

3.2.1. Vegetation Data Analysis. The data collected were used to generate a plot-versus-species matrix (using the percentage cover/abundance values of each species). Cluster analysis using resemblance index and ward's method of hierarchical grouping was performed to identify community groups [18]. Resemblance index was used because it refers to similarity or dissimilarity between samples in terms of species composition. Sample plots that share the same species with the same abundance indicate the highest similarity and the lowest dissimilarity and, therefore, they become one group. Statistical validity of the identified groups was evaluated using multiresponse permutation procedure (MRPP) [19-21]. Both cluster analysis and MRPP were performed using PC-ORD software [18]. The groups were designated as community types and named by most significant indicator species in the group [22].

In this study, the indicator species analysis method proposed by Dufre'n and Legendre [23] was used to identify indicator species. The indicator species are the most characteristic species of each group and present in the majority of the plots belonging to that group (Dufre'n and Legendre [23]).

\subsubsection{Habitat Types Identified from Satellite Data Analysis.} Rapid Eye satellite imagery ( $5 \mathrm{~m}$ resolution) data acquired in 2012 covering all of the Gambella National Park and its surrounding areas was permitted from Applied Science Department of Berlin University. It was first classified by unsupervised classification. The sensor type used in acquiring this imagery for unsupervised classification was multispectral push broom imager and captured five spectral bands (blue $(440-510 \mathrm{~nm})$, green $(520-590 \mathrm{~nm})$, red $(600-700 \mathrm{~nm})$, Red Edge $(690-730 \mathrm{~nm})$, and near-infrared bands (760-850 nm)). ERDAS Imagine 2012 software was used in the preprocessing, pixel-based classification, and postprocessing of the Rapid Eye satellite imagery covering the study area. For the pixel-based classification, the satellite imagery was classified by pixel-based spectral angle mapper (SAM) classifier. The signature file was generated and this involves the training of classes. Areas of interest (AOI) was 


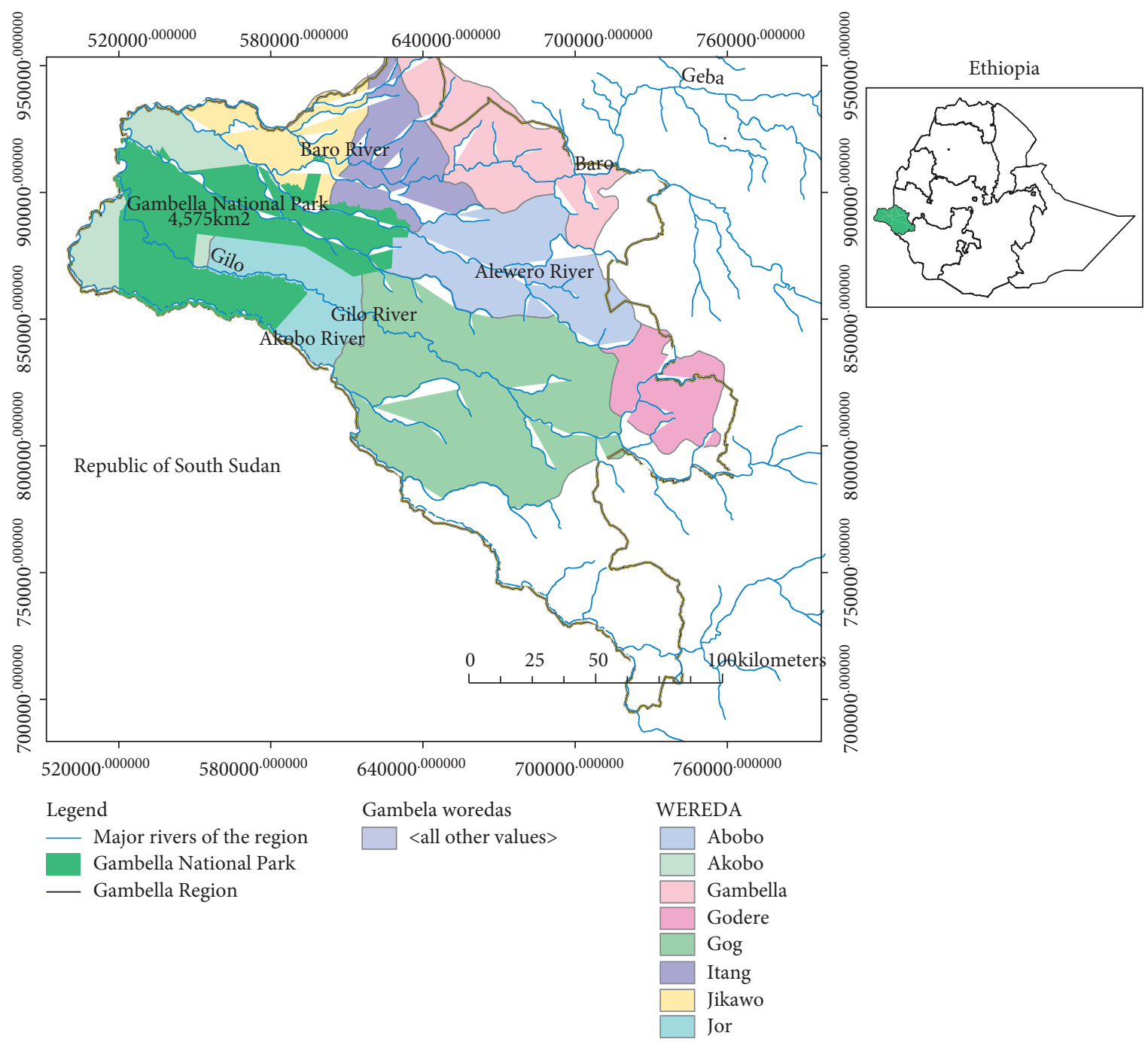

FIgure 1: Location of Gambella National Park within Gambella Regional State.

created and used to train the land cover classes (water body, bare-soil, and vegetation) for every class; random samples were taken across the National Park based on pixel spectra.

The SAM algorithm which is supervised classification approach was then applied. The supervised classification was mainly the ground truth or GPS points. The SAM algorithm was based on the assumption that a single pixel of remote sensing images represents one certain ground cover material, which was uniquely assigned to only one ground cover class. This algorithm was based on the measurement of the spectral similarity between two spectra. The spectral similarity was obtained by considering each spectrum as a vector in $q$-dimensional space, where $q$ is the number of bands.

\section{Results}

4.1. Cluster Classification of Plant Species of Gambella National Park. Six vegetation groups were identified using cluster analysis in combination with multiresponse permutation procedure (MRPP) and the cutoff for this classification was 50\% (Figure 2).
From 80 sample plots, 4 plots were considered as outliers and thus were excluded from the cluster analysis.

The $\mathrm{T}$ value statistic for six groups was $-1.28(P<0.001)$ which indicated the significant different at $P$ value, while the statistic chance-corrected within group agreement was 0.1 . The $T$ statistic is based on Pearson type III distribution. The $P$ value associated with $T$ is determined by numerical integration of type III distribution (Table 1). A statistic is descriptor within a group homogeneity falling between 0 and 1 (Table 1). When the items are identical, $A=1$. Therefore, $A$ statistic is equal to 1 when all items are identical within groups while the delta is equal to $0 . A=0$ when heterogeneity within groups equals expectation by chance.

4.2. Naming of Vegetation Communities by Indicator Species of Study Plant of Gambella National Park. The 6 vegetation communities were named based on indicator values indicated by percent prefect indication value (combining relative abundance and relative frequency) (Tables 2 and 3). The species in bold for each group is one with the maximum indicator values for combining relative abundance and relative frequency. 


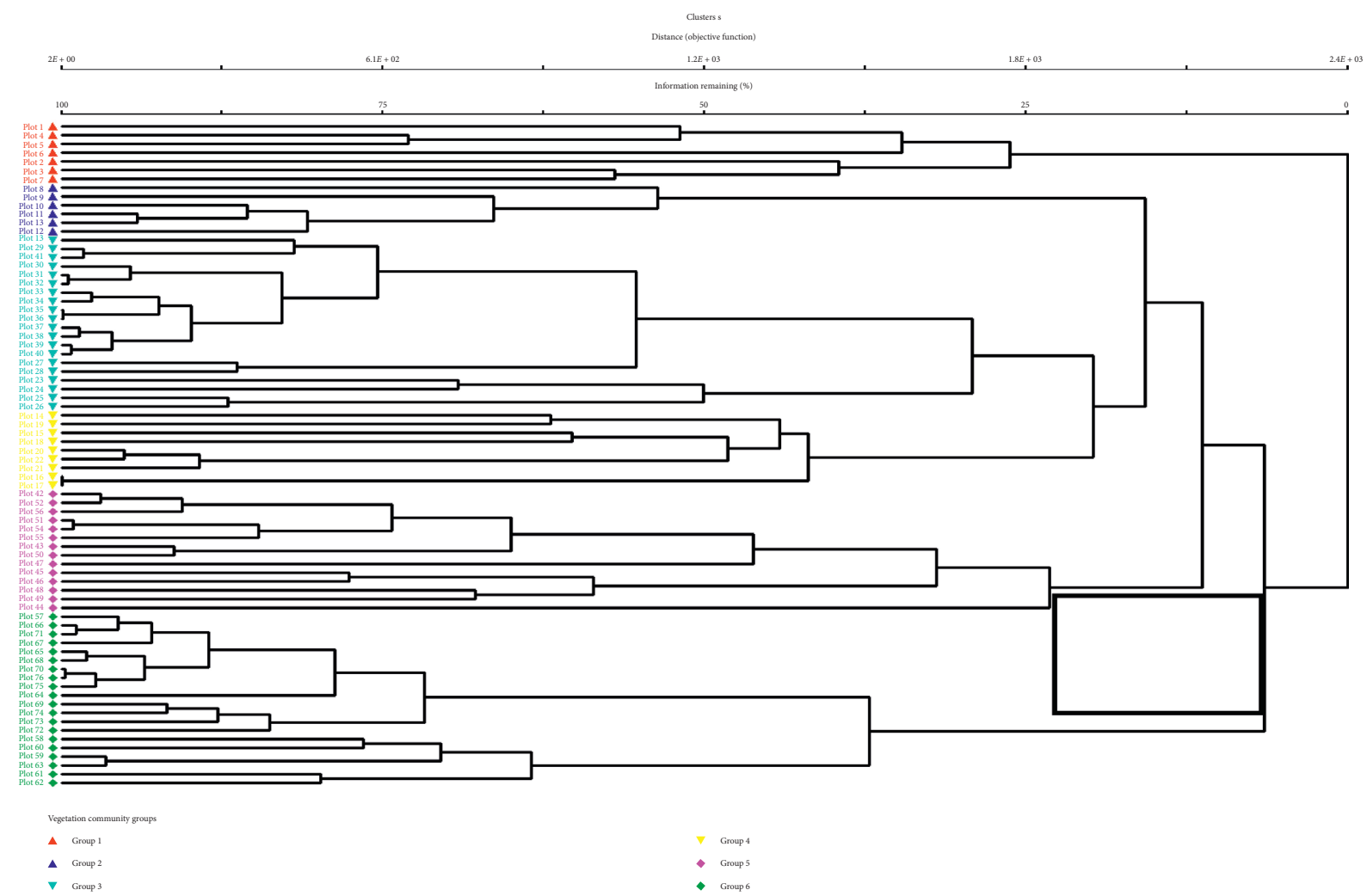

Figure 2: Dendrogram of cluster analysis results of species abundance data of the studied plant species of Gambella National Park. Plots forming the same cluster have similar symbols.

TABle 1: Chance within group agreement.

Chance corrected within group agreement, $A=0.07734179$ $A=1-$ (observed delta/expected delta)

$A_{\max }=1$ when all items are identical within groups $($ delta $=0)$

A statistic is descriptor within a group homogeneity falling between 0 and 1

$A=0$ when heterogeneity within groups equals expectation by chance

$A<0$ with more heterogeneity within groups than expected by chance

Probability of a smaller or equal delta, $P=0.00143541$

Skewness of delta $=$ Pearson type II distribution

Source, 2017 source: result of vegetation analysis, Gatluak, [24]

The maximum indicator values for group 1 species were observed for Combretum collinum (49\%), Combretum molle (47\%), Terminalia brownii (40\%), and Terminalia laxiflora (32\%). This group had formed the vegetation community known as Combretum collinum-Terminalia brownii community. The maximum indicator values for group 2 species were observed for Hyparrhenia rufa (26\%), Oryza barthii (23\%), and Oryza longistaminata (25\%). This group formed Hyparrhenia rufa-Oryza longistaminata community (Table 2), while Cyprerus castaneus (48\%) and Perpyrnuo cypress $(40 \%)$ were species in group 3 with maximum indicator values. These species formed Cyprerus castaneus-Perpyrnuo cypress community.

The maximum indicator values for group 4 species were observed for Ziziphus mucronata (33\%), Acacia senegal
TABle 2: Indicator values (\% of perfect indication based on combining values for relative abundance and relative frequency).

\begin{tabular}{|c|c|c|c|c|c|c|c|}
\hline \multicolumn{8}{|l|}{ Group } \\
\hline Sequence & & 1 & 2 & 3 & 4 & 5 & 6 \\
\hline Identifier & & 1 & 5 & 6 & 8 & 7 & 9 \\
\hline Number of items & & 30 & 11 & 11 & 8 & 8 & 8 \\
\hline \multicolumn{8}{|l|}{ Species MaxGrp } \\
\hline Combretum collinum & 1 & 49 & 0 & 7 & 9 & 4 & 0 \\
\hline Combretum molle & 1 & 47 & 1 & 0 & 0 & 0 & 0 \\
\hline Cyperus castaneus & 3 & 0 & 0 & 48 & 0 & 0 & 0 \\
\hline Hyparrhenia rufa & 2 & 0 & 26 & 0 & 22 & 0 & 0 \\
\hline Oryza barthii & 2 & 0 & 23 & 0 & 1 & 0 & 0 \\
\hline Oryza longistaminata & 2 & 0 & 25 & 0 & 0 & 0 & 0 \\
\hline Perpyrnuo cypress & 3 & 2 & 8 & 40 & 0 & 0 & 0 \\
\hline Terminalia brownii & 1 & 40 & 0 & 0 & 0 & 0 & 0 \\
\hline Terminalia laxiflora & 1 & 32 & 0 & 0 & 0 & 0 & 0 \\
\hline Ziziphus mucronata & 4 & 6 & 3 & 6 & 33 & 0 & \\
\hline
\end{tabular}

MaxGrp = maximum indicator value in group.

(32\%), Acacia polyacantha (22\%), Acacia nilotica (20\%), and Hyparrhenia rufa (22\%). These species formed Ziziphus mucronata-Acacia senegal-Hyparrhenia rufa community. Group 5 was named Acacia nilotica-Acacia bussei community because the maximum indicator values were observed in the Acacia nilotica (30\%) and Acacia bussei (25\%). The maximum indicator values for group 6 were observed in the Balanites aegyptiaca (32\%), Acacia nilotica (22\%), and Acacia asak (21\%). These species formed vegetation community known as Balanites aegyptiaca-Acacia nilotica. 
TABLE 3: Data for vegetation abundance of Gambella National Park.

\begin{tabular}{|c|c|c|}
\hline Plot no. & Species name & Abundance \\
\hline 1 & Combretum aculeatum & 2 \\
\hline 1 & Combretum collinum & 4 \\
\hline 1 & Combretum molle & 4 \\
\hline 1 & Terminalia brownie & 5 \\
\hline 1 & Terminalia laxiflora & 4 \\
\hline 1 & Terminalia macroptera Guill. \& Perr. & 1 \\
\hline 1 & Terminalia schimperiana Hochst. & 1 \\
\hline 1 & Commiphora africana & 1 \\
\hline 1 & Commiphora habessinica & 1 \\
\hline 1 & Albizia malacophylla & 1 \\
\hline 1 & Sterculia africana & 1 \\
\hline 1 & Securidaca longipedunculata & 1 \\
\hline 2 & Combretum collinum & 4 \\
\hline 2 & Combretum molle & 4 \\
\hline 2 & Terminalia brownie & 5 \\
\hline 2 & Terminalia laxiflora & 4 \\
\hline 2 & Terminalia schimperiana Hochst & 1 \\
\hline 2 & Commiphora africana & 1 \\
\hline 2 & Commiphora habessinica & 1 \\
\hline 2 & Cordia africana & 1 \\
\hline 2 & Sterculia africana & 1 \\
\hline 2 & Securidaca longipedunculata & 1 \\
\hline 3 & Combretum collinum & 4 \\
\hline 3 & Combretum molle & 4 \\
\hline 3 & Terminalia brownie & 5 \\
\hline 3 & Terminalia laxiflora & 4 \\
\hline 3 & Terminalia macroptera Guill. \& Perr. & 1 \\
\hline 3 & Terminalia schimperiana Hochst. & 1 \\
\hline 3 & Commiphora africana & 1 \\
\hline \multicolumn{3}{|c|}{ - } \\
\hline 3 & Diospyros mespiliformis & 1 \\
\hline \multicolumn{3}{|c|}{ enop } \\
\hline 3 & Celtis toka & 1 \\
\hline 3 & Securidaca longipedunculata & 1 \\
\hline 4 & Combretum collinum & 4 \\
\hline 4 & Combretum molle & 5 \\
\hline 4 & Terminalia brownie & 4 \\
\hline 4 & Terminalia laxiflora & 3 \\
\hline 4 & Terminalia macroptera Guill. \& Perr. & 1 \\
\hline 4 & Diospyros mespiliformis & 1 \\
\hline 4 & Sarcocephalus latifolius & 1 \\
\hline 4 & Sterculia africana & 1 \\
\hline 5 & Combretum aculeatum & 2 \\
\hline 5 & Combretum collinum & 4 \\
\hline 5 & Combretum molle & 4 \\
\hline 5 & Terminalia brownie & 5 \\
\hline 5 & Terminalia laxiflora & 4 \\
\hline 5 & Terminalia macroptera Guill. \& Perr. & 1 \\
\hline 5 & Terminalia schimperiana Hochst. & 1 \\
\hline 5 & Commiphora africana & 1 \\
\hline 5 & Diospyros mespiliformis & 1 \\
\hline 5 & Sarcocephalus latifolius & 1 \\
\hline 5 & Sterculia africana & 1 \\
\hline 5 & Securidaca longipedunculata & 1 \\
\hline 6 & Combretum collinum & 4 \\
\hline 6 & Combretum molle & 4 \\
\hline 6 & Terminalia brownie & 5 \\
\hline 6 & Terminalia laxiflora & 4 \\
\hline 6 & Terminalia schimperiana Hochst. & 1 \\
\hline 6 & Commiphora africana & 1 \\
\hline 6 & Commiphora habessinica & 1 \\
\hline
\end{tabular}

TABle 3: Continued.

\begin{tabular}{|c|c|c|}
\hline Plot no. & Species name & Abundance \\
\hline 6 & Sarcocephalus latifolius & 1 \\
\hline 6 & Sterculia africana & 1 \\
\hline 6 & Securidaca longipedunculata & 1 \\
\hline 7 & Combretum collinum & 4 \\
\hline 7 & Combretum molle & 4 \\
\hline 7 & Terminalia brownie & 5 \\
\hline 7 & Terminalia laxiflora & 4 \\
\hline 7 & Terminalia macroptera Guill. \& Perr. & 1 \\
\hline 7 & Terminalia schimperiana Hochst. & 1 \\
\hline 7 & Commiphora africana & 1 \\
\hline 7 & Commiphora habessinica & 1 \\
\hline 7 & Sarcocephalus latifolius & 1 \\
\hline 7 & Securidaca longipedunculata & 1 \\
\hline 8 & Combretum collinum & 4 \\
\hline 8 & Combretum molle & 5 \\
\hline 8 & Terminalia brownie & 4 \\
\hline 8 & Terminalia laxiflora & 3 \\
\hline 8 & Terminalia macroptera Guill. \& Perr. & 1 \\
\hline 8 & Commiphora africana & 1 \\
\hline 8 & Sarcocephalus latifolius & 1 \\
\hline 8 & Ficus sycomorus & 1 \\
\hline 9 & Combretum aculeatum & 2 \\
\hline 9 & Combretum collinum & 4 \\
\hline 9 & Combretum molle & 4 \\
\hline 9 & Terminalia brownie & 5 \\
\hline 9 & Terminalia laxiflora & 4 \\
\hline 9 & Terminalia macroptera Guill. \& Perr. & 1 \\
\hline 9 & Terminalia schimperiana Hochst. & 1 \\
\hline 9 & Commiphora africana & 1 \\
\hline 9 & Sclerocarya birrea & 1 \\
\hline 9 & Ficus sycomorus & 1 \\
\hline 9 & Sterculia africana & 1 \\
\hline 9 & Securidaca longipedunculata & 1 \\
\hline 10 & Combretum aculeatum & 3 \\
\hline 10 & Combretum collinum & 3 \\
\hline 10 & Combretum molle & 5 \\
\hline 10 & Terminalia brownie & 4 \\
\hline 10 & Terminalia laxiflora & 4 \\
\hline 10 & Terminalia macroptera Guill. \& Perr. & 1 \\
\hline 10 & Terminalia schimperiana Hochst. & 1 \\
\hline 10 & Commiphora africana & 1 \\
\hline 10 & Commiphora habessinica & 1 \\
\hline 10 & Sarcocephalus latifolius & 1 \\
\hline 10 & Sterculia africana & 1 \\
\hline 10 & Sclerocarya birrea & 1 \\
\hline 11 & Combretum collinum & 4 \\
\hline 11 & Combretum molle & 4 \\
\hline 11 & Terminalia brownie & 5 \\
\hline 11 & Terminalia laxiflora & 4 \\
\hline 11 & Terminalia schimperiana Hochst. & 1 \\
\hline 11 & Commiphora africana & 1 \\
\hline 11 & Commiphora habessinica & 1 \\
\hline 11 & Sclerocarya birrea & 1 \\
\hline 11 & Sterculia africana & 1 \\
\hline 11 & Ficus sycomorus & 1 \\
\hline 12 & Combretum collinum & 4 \\
\hline 12 & Combretum molle & 4 \\
\hline 12 & Terminalia brownie & 5 \\
\hline 12 & Terminalia laxiflora & 4 \\
\hline 12 & Terminalia macroptera Guill. \& Perr. & 1 \\
\hline 12 & Terminalia schimperiana Hochst. & 1 \\
\hline
\end{tabular}


TABle 3: Continued.

\begin{tabular}{|c|c|c|}
\hline Plot no. & Species name & Abundance \\
\hline 12 & Commiphora africana & 1 \\
\hline 12 & Sclerocarya birrea & 1 \\
\hline 12 & Sarcocephalus latifolius & 1 \\
\hline 12 & Securidaca longipedunculata & 1 \\
\hline 13 & Combretum collinum & 4 \\
\hline 13 & Combretum molle & 5 \\
\hline 13 & Terminalia brownie & 4 \\
\hline 13 & Terminalia laxiflora & 3 \\
\hline 13 & Terminalia macroptera Guill. \& Perr. & 1 \\
\hline 13 & Commiphora africana & 1 \\
\hline 13 & Sarcocephalus latifolius & 1 \\
\hline 13 & Sclerocarya birrea & 1 \\
\hline 14 & Combretum aculeatum & 2 \\
\hline 14 & Combretum collinum & 4 \\
\hline 14 & Combretum molle & 4 \\
\hline 14 & Terminalia brownie & 5 \\
\hline 14 & Terminalia laxiflora & 4 \\
\hline 14 & Terminalia macroptera Guill. \& Perr. & 1 \\
\hline 14 & Terminalia schimperiana Hochst. & 1 \\
\hline 14 & Commiphora africana & 1 \\
\hline 14 & Ceiba pentandra & 1 \\
\hline 14 & Ficus vasta Forssk. & 1 \\
\hline 14 & Sterculia africana & 1 \\
\hline 14 & Sclerocarya birrea & 1 \\
\hline 15 & Combretum aculeatum & 3 \\
\hline 15 & Combretum collinum & 3 \\
\hline 15 & Combretum molle & 5 \\
\hline 15 & Terminalia brownie & 4 \\
\hline 15 & Terminalia laxiflora & 4 \\
\hline 15 & Terminalia macroptera Guill. \& Perr. & 1 \\
\hline 15 & Sclerocarya birrea & 1 \\
\hline 15 & Commiphora africana & 1 \\
\hline 15 & Commiphora habessinica & 1 \\
\hline 15 & Sarcocephalus latifolius & 1 \\
\hline 15 & Sterculia africana & 1 \\
\hline 15 & Securidaca longipedunculata & 1 \\
\hline 16 & Combretum collinum & 4 \\
\hline 16 & Combretum molle & 4 \\
\hline 16 & Terminalia brownie & 5 \\
\hline 16 & Terminalia laxiflora & 4 \\
\hline 16 & Terminalia schimperiana Hochst & 1 \\
\hline 16 & Commiphora africana & 1 \\
\hline 16 & Commiphora habessinica & 1 \\
\hline 16 & Salvadora persica & 1 \\
\hline 16 & Sterculia africana & 1 \\
\hline 16 & Ficus vasta Forssk. & 1 \\
\hline 17 & Combretum collinum & 4 \\
\hline 17 & Combretum molle & 4 \\
\hline 17 & Terminalia brownie & 5 \\
\hline 17 & Terminalia laxiflora & 4 \\
\hline 17 & Salvadora persica & 1 \\
\hline 17 & Terminalia schimperiana Hochst. & 1 \\
\hline 17 & Commiphora africana & 1 \\
\hline 17 & Ficus vasta Forssk & 1 \\
\hline 17 & Sarcocephalus latifolius & 1 \\
\hline 18 & Securidaca longipedunculata & 1 \\
\hline 18 & Combretum collinum & 4 \\
\hline 18 & Combretum molle & 5 \\
\hline 18 & Terminalia brownie & 4 \\
\hline 18 & Terminalia laxiflora & 3 \\
\hline 18 & Terminalia macroptera Guill. \& Perr. & 1 \\
\hline
\end{tabular}

TABle 3: Continued.

\begin{tabular}{|c|c|c|}
\hline Plot no. & Species name & Abundance \\
\hline 18 & Commiphora africana & 1 \\
\hline 18 & Sarcocephalus latifolius & 1 \\
\hline 18 & Sterculia africana & 1 \\
\hline 19 & Terminalia brownie & 5 \\
\hline 19 & Capparis erythrocarpus & 1 \\
\hline 19 & Terminalia laxiflora & 4 \\
\hline 19 & Salvadora persica & 1 \\
\hline 19 & Tamarindus indica & 2 \\
\hline 19 & Acacia oerfota & 1 \\
\hline 19 & Maytenus senegalensis & 1 \\
\hline 19 & Ziziphus mucronata & 2 \\
\hline 20 & Terminalia brownie & 4 \\
\hline 20 & Capparis erythrocarpus & 1 \\
\hline 20 & Terminalia laxiflora & 4 \\
\hline 20 & Boswellia rivae & 1 \\
\hline 20 & Tamarindus indica & 2 \\
\hline 20 & Acacia oerfota & 1 \\
\hline 20 & Maytenus senegalensis & 1 \\
\hline 20 & Grewia bicolor & 2 \\
\hline 20 & Terminalia brownie & 5 \\
\hline 20 & Capparis erythrocarpus & 1 \\
\hline 20 & Terminalia laxiflora & 4 \\
\hline 20 & Boswellia rivae & 1 \\
\hline 20 & Tamarindus indica & 2 \\
\hline 20 & Acacia oerfota & 1 \\
\hline 20 & Grewia bicolor & 1 \\
\hline 20 & Ziziphus mucronata & 1 \\
\hline 21 & Terminalia brownie & 4 \\
\hline 21 & Capparis erythrocarpus & 1 \\
\hline 21 & Terminalia laxiflora & 4 \\
\hline 21 & Boswellia rivae & 1 \\
\hline 21 & Tamarindus indica & 2 \\
\hline 21 & Grewia bicolor & 1 \\
\hline 21 & Maytenus senegalensis & 1 \\
\hline 21 & Ziziphus mucronata & 1 \\
\hline 23 & Terminalia brownie & 5 \\
\hline 23 & Terminalia laxiflora & 5 \\
\hline 23 & Boswellia rivae & 1 \\
\hline 23 & Tamarindus indica & 1 \\
\hline 23 & Maytenus senegalensis & 1 \\
\hline 23 & Ziziphus mucronata & 1 \\
\hline 24 & Terminalia brownie & 5 \\
\hline 24 & Capparis erythrocarpus & 1 \\
\hline 24 & Terminalia laxiflora & 4 \\
\hline 24 & Boswellia rivae & 1 \\
\hline 24 & Tamarindus indica & 2 \\
\hline 24 & Acacia oerfota & 1 \\
\hline 24 & Maytenus senegalensis & 1 \\
\hline 24 & Ziziphus mucronata & 2 \\
\hline 25 & Terminalia brownie & 4 \\
\hline 25 & Capparis erythrocarpus & 1 \\
\hline 25 & Terminalia laxiflora & 4 \\
\hline 25 & Boswellia rivae & 1 \\
\hline 25 & Tamarindus indica & 2 \\
\hline 25 & Acacia oerfota & 1 \\
\hline 25 & Maytenus senegalensis & 1 \\
\hline 25 & Ziziphus mucronata & 2 \\
\hline 26 & Terminalia brownie & 5 \\
\hline 26 & Capparis erythrocarpus & 1 \\
\hline 26 & Terminalia laxiflora & 4 \\
\hline 26 & Boswellia rivae & 1 \\
\hline
\end{tabular}


TABle 3: Continued.

\begin{tabular}{|c|c|c|}
\hline Plot no. & Species name & Abundance \\
\hline 26 & Tamarindus indica & 2 \\
\hline 26 & Acacia oerfota & 1 \\
\hline 26 & Maytenus senegalensis & 1 \\
\hline 26 & Ziziphus mucronata & 1 \\
\hline 27 & Terminalia brownie & 4 \\
\hline 27 & Capparis erythrocarpus & 1 \\
\hline 27 & Terminalia laxiflora & 4 \\
\hline 27 & Boswellia rivae & 1 \\
\hline 27 & Tamarindus indica & 2 \\
\hline 27 & Acacia oerfota & 1 \\
\hline 27 & Maytenus senegalensis & 1 \\
\hline 27 & Ziziphus mucronata & 1 \\
\hline 28 & Terminalia brownie & 5 \\
\hline 28 & Terminalia laxiflora & 5 \\
\hline 28 & Boswellia rivae & 1 \\
\hline 28 & Tamarindus indica & 1 \\
\hline 28 & Maytenus senegalensis & 1 \\
\hline 28 & Ziziphus mucronata & 1 \\
\hline 29 & Terminalia brownie & 4 \\
\hline 29 & Terminalia laxiflora & 5 \\
\hline 29 & Boswellia rivae & 1 \\
\hline 29 & Tamarindus indica & 2 \\
\hline 29 & Maytenus senegalensis & 1 \\
\hline 29 & Ziziphus mucronata & 1 \\
\hline 30 & Terminalia brownie & 5 \\
\hline 30 & Terminalia laxiflora & 5 \\
\hline 30 & Boswellia rivae & 1 \\
\hline 30 & Tamarindus indica & 1 \\
\hline 30 & Ricinus communis & 1 \\
\hline 30 & Ziziphus mucronata & 1 \\
\hline 31 & Terminalia brownie & 5 \\
\hline 31 & Terminalia laxiflora & 5 \\
\hline 31 & Boswellia rivae & 1 \\
\hline 31 & Tamarindus indica & 1 \\
\hline 31 & Maytenus senegalensis & 1 \\
\hline 31 & Ricinus communis & 1 \\
\hline 32 & Tamarindus indica & 5 \\
\hline 32 & Terminalia brownie & 4 \\
\hline 32 & Terminalia laxiflora & 3 \\
\hline 32 & Steganotaenia araliacea & 1 \\
\hline 32 & Strychnos innocua & 1 \\
\hline 32 & Sterculia africana & 2 \\
\hline 32 & Vitellaria paradoxa & 2 \\
\hline 33 & Tamarindus indica & 5 \\
\hline 33 & Terminalia brownie & 4 \\
\hline 33 & Terminalia laxiflora & 4 \\
\hline 33 & Ricinus communis & 1 \\
\hline 33 & Strychnos innocua & 1 \\
\hline 33 & Sterculia africana & 1 \\
\hline 33 & Vitellaria paradoxa & 2 \\
\hline 34 & Tamarindus indica & 4 \\
\hline 34 & Terminalia brownie & 4 \\
\hline 34 & Terminalia laxiflora & 3 \\
\hline 34 & Steganotaenia araliacea & 1 \\
\hline 34 & Ricinus communis & 1 \\
\hline 34 & Sterculia africana & 1 \\
\hline 34 & Vitellaria paradoxa & 1 \\
\hline 35 & Tamarindus indica & 5 \\
\hline 35 & Terminalia brownie & 4 \\
\hline 35 & Terminalia laxiflora & 4 \\
\hline 35 & Steganotaenia araliacea & 1 \\
\hline
\end{tabular}

TABle 3: Continued.

\begin{tabular}{|c|c|c|}
\hline Plot no. & Species name & Abundance \\
\hline 35 & Strychnos innocua & 1 \\
\hline 35 & Sterculia africana & 1 \\
\hline 35 & Vitellaria paradoxa & 2 \\
\hline 36 & Tamarindus indica & 5 \\
\hline 36 & Terminalia brownie & 4 \\
\hline 36 & Terminalia laxiflora & 4 \\
\hline 36 & Steganotaenia araliacea & 1 \\
\hline 36 & Grewia mollis A.Juss. & 1 \\
\hline 36 & Sterculia africana & 2 \\
\hline 36 & Vitellaria paradoxa & 1 \\
\hline 37 & Tamarindus indica & 5 \\
\hline 37 & Terminalia brownie & 4 \\
\hline 37 & Terminalia laxiflora & 4 \\
\hline 37 & Steganotaenia araliacea & 1 \\
\hline 37 & Strychnos innocua & 1 \\
\hline 37 & Sterculia africana & 1 \\
\hline 37 & Vitellaria paradoxa & 2 \\
\hline 38 & Tamarindus indica & 4 \\
\hline 38 & Terminalia brownie & 4 \\
\hline 38 & Terminalia laxiflora & 3 \\
\hline 38 & Grewia mollis A.Juss. & 1 \\
\hline 38 & Strychnos innocua & 1 \\
\hline 38 & Sterculia africana & 1 \\
\hline 38 & Vitellaria paradoxa & 1 \\
\hline 39 & Tamarindus indica & 5 \\
\hline 39 & Terminalia brownie & 4 \\
\hline 39 & Terminalia laxiflora & 4 \\
\hline 39 & Steganotaenia araliacea & 1 \\
\hline 39 & Strychnos innocua & 1 \\
\hline 39 & Sterculia africana & 2 \\
\hline 39 & Vitellaria paradoxa & 1 \\
\hline 40 & Balanites aegyptiaca & 5 \\
\hline 40 & Acacia seyal & 3 \\
\hline 40 & Acacia senegal & 3 \\
\hline 40 & Acacia polyacantha & 2 \\
\hline 40 & Acacia tortilis & 2 \\
\hline 40 & Acacia oerfota & 2 \\
\hline 40 & Tamarindus indica & 1 \\
\hline 40 & Flueggea virosa & 1 \\
\hline 40 & Ziziphus mucronata & 1 \\
\hline 40 & Piliostigma thonningii & 1 \\
\hline 41 & Balanites aegyptiaca & 5 \\
\hline 41 & Acacia seyal & 3 \\
\hline 41 & Acacia senegal & 3 \\
\hline 41 & Acacia polyacantha & 2 \\
\hline 41 & Acacia tortilis & 2 \\
\hline 41 & Acacia oerfota & 2 \\
\hline 41 & Tamarindus indica & 1 \\
\hline 41 & Flueggea virosa & 1 \\
\hline 41 & Saba Florida (Benth.) Bullock & 1 \\
\hline 42 & Balanites aegyptiaca & 5 \\
\hline 42 & Acacia seyal & 4 \\
\hline 42 & Acacia senegal & 3 \\
\hline 42 & Acacia polyacantha & 2 \\
\hline 42 & Acacia tortilis & 2 \\
\hline 42 & Acacia oerfota & 2 \\
\hline 42 & Flueggea virosa & 1 \\
\hline 42 & Grewia mollis A.Juss. & 1 \\
\hline 42 & Piliostigma thonningii & 1 \\
\hline 43 & Balanites aegyptiaca & 5 \\
\hline 43 & Acacia seyal & 3 \\
\hline
\end{tabular}


Table 3: Continued.

\begin{tabular}{|c|c|c|}
\hline Plot no. & Species name & Abundance \\
\hline 43 & Acacia senegal & 3 \\
\hline 43 & Acacia polyacantha & 2 \\
\hline 43 & Acacia tortilis & 2 \\
\hline 43 & Acacia oerfota & 2 \\
\hline 43 & Tamarindus indica & 1 \\
\hline 43 & Flueggea virosa & 1 \\
\hline 43 & Ziziphus mucronata & 1 \\
\hline 43 & Saba Florida (Benth.) Bullock & 1 \\
\hline 44 & Balanites aegyptiaca & 5 \\
\hline 44 & Acacia seyal & 3 \\
\hline 44 & Acacia senegal & 3 \\
\hline 44 & Acacia polyacantha & 2 \\
\hline 44 & Acacia tortilis & 2 \\
\hline 44 & Acacia oerfota & 2 \\
\hline 44 & Tamarindus indica & 1 \\
\hline 44 & Piliostigma thonningii & 1 \\
\hline 44 & Saba Florida (Benth.) Bullock & 1 \\
\hline 45 & Balanites aegyptiaca & 4 \\
\hline 45 & Acacia seyal & 4 \\
\hline 45 & Acacia senegal & 3 \\
\hline 45 & Acacia polyacantha & 2 \\
\hline 45 & Acacia tortilis & 3 \\
\hline 45 & Acacia oerfota & 2 \\
\hline 45 & Tamarindus indica & 1 \\
\hline 45 & Grewia mollis A.Juss. & 1 \\
\hline 45 & Capparis tomentosa & 1 \\
\hline 46 & Balanites aegyptiaca & 5 \\
\hline 46 & Acacia seyal & 4 \\
\hline 46 & Acacia senegal & 3 \\
\hline 46 & Acacia polyacantha & 2 \\
\hline 46 & Acacia tortilis & 2 \\
\hline 46 & Piliostigma thonningii & 2 \\
\hline 46 & Flueggea virosa & 1 \\
\hline 46 & Ziziphus mucronata & 1 \\
\hline 46 & Capparis tomentosa & 1 \\
\hline 47 & Balanites aegyptiaca & 5 \\
\hline 47 & Acacia seyal & 3 \\
\hline 47 & Acacia senegal & 3 \\
\hline 47 & Acacia polyacantha & 2 \\
\hline 47 & Acacia tortilis & 2 \\
\hline 47 & Piliostigma thonningii & 2 \\
\hline 47 & Tamarindus indica & 1 \\
\hline 47 & Flueggea virosa & 1 \\
\hline 47 & Ziziphus mucronata & 1 \\
\hline 48 & Balanites aegyptiaca & 4 \\
\hline 48 & Acacia seyal & 4 \\
\hline 48 & Acacia senegal & 3 \\
\hline 48 & Acacia polyacantha & 2 \\
\hline 48 & Acacia tortilis & 3 \\
\hline 48 & Acacia oerfota & 2 \\
\hline 48 & Tamarindus indica & 1 \\
\hline 48 & Flueggea virosa & 1 \\
\hline 48 & Grewia tenax (Forssk.) Fiori & 1 \\
\hline 49 & Balanites aegyptiaca & 5 \\
\hline 49 & Acacia seyal & 4 \\
\hline 49 & Acacia senegal & 3 \\
\hline 49 & Acacia polyacantha & 2 \\
\hline 49 & Acacia tortilis & 2 \\
\hline 49 & Acacia oerfota & 2 \\
\hline 49 & Flueggea virosa & 1 \\
\hline 49 & Grewia tenax (Forssk.) Fiori & 1 \\
\hline
\end{tabular}

TABle 3: Continued.

\begin{tabular}{|c|c|c|}
\hline Plot no. & Species name & Abundance \\
\hline 49 & Capparis tomentosa & 1 \\
\hline 50 & Balanites aegyptiaca & 5 \\
\hline 50 & Acacia seyal & 3 \\
\hline 50 & Acacia senegal & 3 \\
\hline 50 & Acacia polyacantha & 2 \\
\hline 50 & Acacia tortilis & 2 \\
\hline 50 & Acacia oerfota & 2 \\
\hline 50 & Grewia tenax (Forssk.) Fiori & 1 \\
\hline 50 & Flueggea virosa & 1 \\
\hline 50 & Ziziphus mucronata & 1 \\
\hline 50 & Balanites aegyptiaca & 4 \\
\hline 50 & Acacia seyal & 3 \\
\hline 50 & Acacia senegal & 3 \\
\hline 50 & Acacia polyacantha & 2 \\
\hline 50 & Acacia tortilis & 3 \\
\hline 50 & Acacia oerfota & 2 \\
\hline 50 & Tamarindus indica & 1 \\
\hline 50 & Flueggea virosa & 1 \\
\hline 50 & Capparis tomentosa & 1 \\
\hline 51 & Balanites aegyptiaca & 5 \\
\hline 51 & Acacia seyal & 4 \\
\hline 51 & Acacia senegal & 3 \\
\hline 51 & Acacia polyacantha & 2 \\
\hline 51 & Acacia tortilis & 2 \\
\hline 51 & Acacia oerfota & 2 \\
\hline 51 & Flueggea virosa & 1 \\
\hline 51 & Ziziphus mucronata & 1 \\
\hline 51 & Capparis tomentosa & 1 \\
\hline 52 & Balanites aegyptiaca & 5 \\
\hline 52 & Acacia seyal & 3 \\
\hline 52 & Acacia senegal & 3 \\
\hline 52 & Acacia polyacantha & 2 \\
\hline 52 & Acacia tortilis & 2 \\
\hline 52 & Acacia oerfota & 2 \\
\hline 52 & Tamarindus indica & 1 \\
\hline 52 & Flueggea virosa & 1 \\
\hline 52 & Ziziphus mucronata & 1 \\
\hline 52 & Capparis tomentosa & 1 \\
\hline 53 & Balanites aegyptiaca & 5 \\
\hline 53 & Acacia seyal & 3 \\
\hline 53 & Acacia senegal & 3 \\
\hline 53 & Acacia polyacantha & 2 \\
\hline 53 & Acacia tortilis & 2 \\
\hline 53 & Acacia oerfota & 2 \\
\hline 53 & Tamarindus indica & 1 \\
\hline 53 & Flueggea virosa & 1 \\
\hline 53 & Ziziphus mucronata & 1 \\
\hline 54 & Balanites aegyptiaca & 4 \\
\hline 54 & Acacia seyal & 4 \\
\hline 54 & Acacia senegal & 2 \\
\hline 54 & Acacia polyacantha & 2 \\
\hline 54 & Acacia tortilis & 3 \\
\hline 54 & Acacia oerfota & 2 \\
\hline 54 & Tamarindus indica & 1 \\
\hline 54 & Flueggea virosa & 1 \\
\hline 54 & Capparis tomentosa & 1 \\
\hline 55 & Balanites aegyptiaca & 5 \\
\hline 55 & Acacia seyal & 4 \\
\hline 55 & Acacia senegal & 3 \\
\hline 55 & Acacia polyacantha & 2 \\
\hline 55 & Acacia tortilis & 2 \\
\hline
\end{tabular}


Table 3: Continued.

\begin{tabular}{|c|c|c|}
\hline Plot no. & Species name & Abundance \\
\hline 55 & Acacia oerfota & 2 \\
\hline 55 & Flueggea virosa & 1 \\
\hline 55 & Ziziphus mucronata & 1 \\
\hline 55 & Capparis tomentosa & 1 \\
\hline 56 & Balanites aegyptiaca & 5 \\
\hline 56 & Acacia seyal & 3 \\
\hline 56 & Acacia senegal & 3 \\
\hline 56 & Acacia polyacantha & 2 \\
\hline 56 & Acacia tortilis & 2 \\
\hline 56 & Acacia oerfota & 2 \\
\hline 56 & Tamarindus indica & 1 \\
\hline 56 & Flueggea virosa & 1 \\
\hline 56 & Ziziphus mucronata & 1 \\
\hline 56 & Capparis tomentosa & 1 \\
\hline 57 & Balanites aegyptiaca & 5 \\
\hline 57 & Acacia seyal & 3 \\
\hline 57 & Acacia senegal & 3 \\
\hline 57 & Acacia polyacantha & 2 \\
\hline 57 & Acacia tortilis & 2 \\
\hline 57 & Acacia oerfota & 2 \\
\hline 57 & Tamarindus indica & 1 \\
\hline 57 & Flueggea virosa & 1 \\
\hline 57 & Ziziphus mucronata & 1 \\
\hline 58 & Balanites aegyptiaca & 4 \\
\hline 58 & Acacia seyal & 4 \\
\hline 58 & Acacia senegal & 3 \\
\hline 58 & Acacia polyacantha & 2 \\
\hline 58 & Acacia tortilis & 3 \\
\hline 58 & Acacia oerfota & 2 \\
\hline 58 & Tamarindus indica & 1 \\
\hline 58 & Flueggea virosa & 1 \\
\hline 58 & Capparis tomentosa & 1 \\
\hline 59 & Balanites aegyptiaca & 5 \\
\hline 59 & Acacia seyal & 3 \\
\hline 59 & Acacia senegal & 3 \\
\hline 59 & Acacia polyacantha & 2 \\
\hline 59 & Acacia tortilis & 2 \\
\hline 59 & Acacia oerfota & 2 \\
\hline 59 & Tamarindus indica & 1 \\
\hline 59 & Flueggea virosa & 1 \\
\hline 59 & Ziziphus mucronata & 1 \\
\hline 59 & Capparis tomentosa & 1 \\
\hline 60 & Balanites aegyptiaca & 5 \\
\hline 60 & Acacia seyal & 3 \\
\hline 60 & Acacia senegal & 3 \\
\hline 60 & Acacia polyacantha & 2 \\
\hline 60 & Acacia tortilis & 2 \\
\hline 60 & Acacia oerfota & 2 \\
\hline 60 & Tamarindus indica & 1 \\
\hline 60 & Flueggea virosa & 1 \\
\hline 60 & Ziziphus mucronata & 1 \\
\hline 61 & Balanites aegyptiaca & 4 \\
\hline 61 & Acacia seyal & 4 \\
\hline 61 & Acacia senegal & 3 \\
\hline 61 & Acacia polyacantha & 2 \\
\hline 61 & Acacia tortilis & 3 \\
\hline 61 & Acacia oerfota & 2 \\
\hline 61 & Tamarindus indica & 1 \\
\hline 61 & Flueggea virosa & 1 \\
\hline 61 & Capparis tomentosa & 1 \\
\hline 62 & Balanites aegyptiaca & 5 \\
\hline
\end{tabular}

TABle 3: Continued.

\begin{tabular}{|c|c|c|}
\hline Plot no. & Species name & Abundance \\
\hline 62 & Acacia seyal & 4 \\
\hline 62 & Acacia senegal & 3 \\
\hline 62 & Acacia polyacantha & 2 \\
\hline 62 & Acacia tortilis & 2 \\
\hline 62 & Acacia oerfota & 2 \\
\hline 62 & Flueggea virosa & 1 \\
\hline 62 & Ziziphus mucronata & 1 \\
\hline 62 & Capparis tomentosa & 1 \\
\hline 63 & Balanites aegyptiaca & 5 \\
\hline 63 & Acacia seyal & 3 \\
\hline 63 & Acacia senegal & 3 \\
\hline 63 & Acacia polyacantha & 2 \\
\hline 63 & Acacia tortilis & 2 \\
\hline 63 & Acacia oerfota & 2 \\
\hline 63 & Tamarindus indica & 1 \\
\hline 63 & Flueggea virosa & 1 \\
\hline 63 & Ziziphus mucronata & 1 \\
\hline 63 & Capparis tomentosa & 1 \\
\hline 64 & Balanites aegyptiaca & 5 \\
\hline 64 & Acacia seyal & 3 \\
\hline 64 & Acacia senegal & 3 \\
\hline 64 & Acacia polyacantha & 2 \\
\hline 64 & Acacia tortilis & 2 \\
\hline 64 & Acacia oerfota & 2 \\
\hline 64 & Tamarindus indica & 1 \\
\hline 64 & Flueggea virosa & 1 \\
\hline 64 & Ziziphus mucronata & 1 \\
\hline 65 & Balanites aegyptiaca & 4 \\
\hline 65 & Acacia seyal & 4 \\
\hline 65 & Acacia senegal & 3 \\
\hline 65 & Acacia polyacantha & 2 \\
\hline 65 & Acacia tortilis & 3 \\
\hline 65 & Acacia oerfota & 2 \\
\hline 65 & Tamarindus indica & 1 \\
\hline 65 & Flueggea virosa & 1 \\
\hline 65 & Capparis tomentosa & 1 \\
\hline 66 & Balanites aegyptiaca & 5 \\
\hline 66 & Acacia seyal & 3 \\
\hline 66 & Acacia senegal & 3 \\
\hline 66 & Acacia polyacantha & 2 \\
\hline 66 & Acacia tortilis & 2 \\
\hline 66 & Acacia oerfota & 2 \\
\hline 66 & Tamarindus indica & 1 \\
\hline 66 & Flueggea virosa & 1 \\
\hline 66 & Ziziphus mucronata & 1 \\
\hline 66 & Capparis tomentosa & 1 \\
\hline 67 & Balanites aegyptiaca & 5 \\
\hline 67 & Acacia seyal & 3 \\
\hline 67 & Acacia senegal & 3 \\
\hline 67 & Acacia polyacantha & 2 \\
\hline 67 & Acacia tortilis & 2 \\
\hline 67 & Acacia oerfota & 2 \\
\hline 67 & Tamarindus indica & 1 \\
\hline 67 & Flueggea virosa & 1 \\
\hline 67 & Ziziphus mucronata & 1 \\
\hline 68 & Balanites aegyptiaca & 4 \\
\hline 68 & Acacia seyal & 3 \\
\hline 68 & Acacia senegal & 3 \\
\hline 68 & Acacia polyacantha & 2 \\
\hline 68 & Acacia tortilis & 3 \\
\hline 68 & Acacia oerfota & 2 \\
\hline
\end{tabular}


TABle 3: Continued.

\begin{tabular}{|c|c|c|}
\hline Plot no. & Species name & Abundance \\
\hline 68 & Tamarindus indica & 1 \\
\hline 68 & Flueggea virosa & 1 \\
\hline 68 & Capparis tomentosa & 1 \\
\hline 69 & Balanites aegyptiaca & 5 \\
\hline 69 & Acacia seyal & 4 \\
\hline 69 & Acacia senegal & 3 \\
\hline 69 & Acacia polyacantha & 2 \\
\hline 69 & Acacia tortilis & 2 \\
\hline 69 & Acacia oerfota & 2 \\
\hline 69 & Flueggea virosa & 1 \\
\hline 69 & Ziziphus mucronata & 1 \\
\hline 69 & Capparis tomentosa & 1 \\
\hline 70 & Balanites aegyptiaca & 5 \\
\hline 70 & Acacia seyal & 3 \\
\hline 70 & Acacia senegal & 3 \\
\hline 70 & Acacia polyacantha & 2 \\
\hline 70 & Acacia tortilis & 2 \\
\hline 70 & Acacia oerfota & 2 \\
\hline 70 & Tamarindus indica & 1 \\
\hline 70 & Flueggea virosa & 1 \\
\hline 70 & Ziziphus mucronata & 1 \\
\hline 71 & Balanites aegyptiaca & 4 \\
\hline 71 & Acacia seyal & 4 \\
\hline 71 & Acacia senegal & 3 \\
\hline 71 & Acacia polyacantha & 2 \\
\hline 71 & Acacia tortilis & 3 \\
\hline 71 & Acacia oerfota & 2 \\
\hline 71 & Tamarindus indica & 1 \\
\hline 71 & Flueggea virosa & 1 \\
\hline 71 & Capparis tomentosa & 1 \\
\hline 72 & Balanites aegyptiaca & 5 \\
\hline 72 & Acacia seyal & 4 \\
\hline 72 & Acacia senegal & 3 \\
\hline 72 & Acacia polyacantha & 2 \\
\hline 72 & Acacia tortilis & 2 \\
\hline 72 & Acacia oerfota & 2 \\
\hline 72 & Flueggea virosa & 1 \\
\hline 72 & Ziziphus mucronata & 1 \\
\hline 72 & Capparis tomentosa & 1 \\
\hline 73 & Balanites aegyptiaca & 5 \\
\hline 73 & Acacia seyal & 3 \\
\hline 73 & Acacia senegal & 3 \\
\hline 73 & Acacia polyacantha & 2 \\
\hline 73 & Acacia tortilis & 2 \\
\hline 73 & Acacia oerfota & 2 \\
\hline 73 & Tamarindus indica & 1 \\
\hline 73 & Flueggea virosa & 1 \\
\hline 73 & Ziziphus mucronata & 1 \\
\hline 74 & Balanites aegyptiaca & 5 \\
\hline 74 & Acacia seyal & 3 \\
\hline 74 & Acacia senegal & 3 \\
\hline 74 & Acacia polyacantha & 2 \\
\hline 74 & Acacia tortilis & 2 \\
\hline 74 & Acacia oerfota & 2 \\
\hline 74 & Tamarindus indica & 1 \\
\hline 74 & Flueggea virosa & 1 \\
\hline 74 & Ziziphus mucronata & 1 \\
\hline 75 & Balanites aegyptiaca & 4 \\
\hline 75 & Acacia seyal & 3 \\
\hline 75 & Acacia senegal & 3 \\
\hline 75 & Acacia polyacantha & 2 \\
\hline
\end{tabular}

TABle 3: Continued.

\begin{tabular}{|c|c|c|}
\hline Plot no. & Species name & Abundance \\
\hline 75 & Acacia tortilis & 3 \\
\hline 75 & Acacia oerfota & 2 \\
\hline 75 & Tamarindus indica & 1 \\
\hline 75 & Flueggea virosa & 1 \\
\hline 75 & Capparis tomentosa & 1 \\
\hline 76 & Balanites aegyptiaca & 5 \\
\hline 76 & Acacia senegal & 3 \\
\hline 76 & Acacia polyacantha & 2 \\
\hline 76 & Acacia tortilis & 2 \\
\hline 76 & Acacia oerfota & 2 \\
\hline 76 & Flueggea virosa & 1 \\
\hline 76 & Ziziphus mucronata & 1 \\
\hline 77 & Balanites aegyptiaca & 5 \\
\hline 77 & Acacia seyal & 3 \\
\hline 77 & Acacia senegal & 3 \\
\hline 77 & Acacia polyacantha & 2 \\
\hline 77 & Acacia tortilis & 2 \\
\hline 77 & Acacia oerfota & 2 \\
\hline 77 & Tamarindus indica & 1 \\
\hline 77 & Flueggea virosa & 1 \\
\hline 77 & Ziziphus mucronata & 1 \\
\hline 78 & Balanites aegyptiaca & 4 \\
\hline 78 & Acacia seyal & 3 \\
\hline 78 & Acacia senegal & 3 \\
\hline 78 & Acacia polyacantha & 2 \\
\hline 78 & Acacia tortilis & 3 \\
\hline 78 & Acacia oerfota & 2 \\
\hline 78 & Tamarindus indica & 1 \\
\hline 78 & Flueggea virosa & 1 \\
\hline 78 & Capparis tomentosa & 1 \\
\hline 79 & Balanites aegyptiaca & 5 \\
\hline 79 & Acacia seyal & 4 \\
\hline 79 & Acacia senegal & 3 \\
\hline 79 & Acacia polyacantha & 2 \\
\hline 79 & Acacia tortilis & 2 \\
\hline 79 & Acacia oerfota & 2 \\
\hline 80 & Balanites aegyptiaca & 5 \\
\hline 80 & Acacia seyal & 3 \\
\hline 80 & Acacia Senegal & 3 \\
\hline 80 & Acacia polyacantha & 2 \\
\hline 80 & Acacia tortilis & 2 \\
\hline 80 & Acacia oerfota & 2 \\
\hline 80 & Tamarindus indica & 1 \\
\hline 80 & Flueggea virosa & 1 \\
\hline 80 & Ziziphus mucronata & 1 \\
\hline 80 & Capparis tomentosa & 1 \\
\hline
\end{tabular}

Source: vegetation raw data for Ph.D. thesis, Gatluak [24].

4.3. Habitat Types of Gambella National Park. The Rapid Eye image classified the Gambella National Park into five major habitat types which included woodland, wooded grassland, savanna, grassland, and wetlands (Figure 3). Other minor habitat types classified on the map were temporary burned, water body, and rivers.

The woodland comprised three vegetation community types. These were Combretum collinum-Terminalia brownii, Acacia nilotica-Acacia bussei, and Balanites aegyptiaca-Acacia nilotica communities while Ziziphus mucronata-Acacia senegal-Hyparrhenia rufa community was found in wooded 


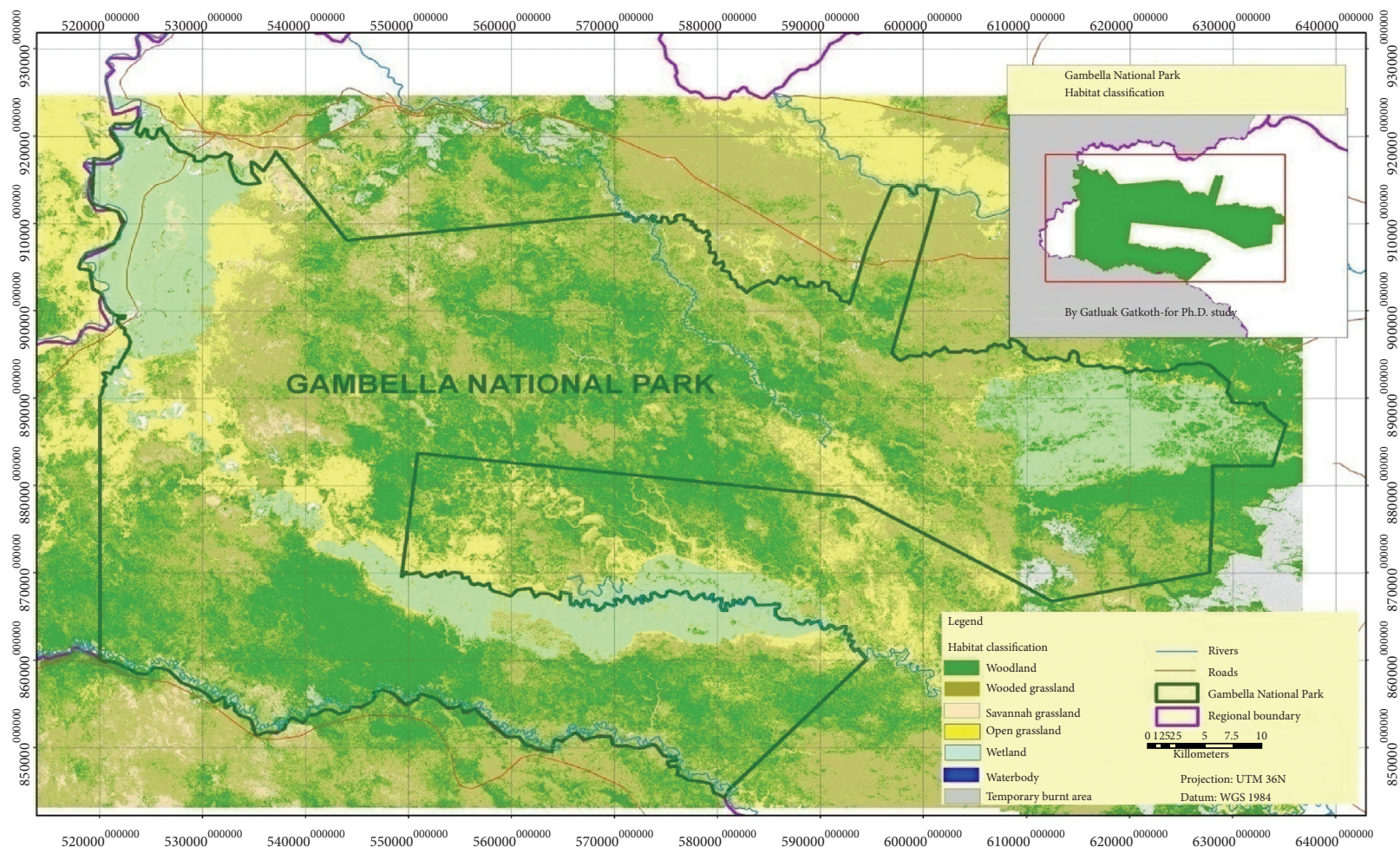

FIgure 3: Habitat types of Gambella National Park.

Table 4: Area covered by each habitat type.

\begin{tabular}{lccc}
\hline No. & Habitat classification & Area in $\mathrm{km}^{2}$ & Percentage \\
\hline 1 & Grassland & 395.50 & 6.74 \\
2 & Savannah & 64.63 & 1.52 \\
5 & Wet land & 1650.04 & 14.27 \\
4 & Wooded grassland & 1716.50 & 36.45 \\
3 & Woodland & & 37.92 \\
6 & Others & 0.16 & 0.00 \\
6.1 & Settlements/roads & 23.15 & 0.50 \\
6.2 & Temporary burned & 27.15 & 0.60 \\
6.3 & Water body & $\mathbf{4 , 5 2 7 . 1 2}$ & $\mathbf{1 0 0}$ \\
\hline
\end{tabular}

grassland habitat of the park. Hyparrhenia rufa-Oryza longistaminata and Cyperus castaneus-Perpyrnuo cypress community was found in open grassland and wetland, respectively.

The classification of habitats for Gambella National Park had also shown that woodland in Gambella National Park cover an area of $1,716.50$ square kilometers (37.92\%) of the land cover of the park followed by wooded grassland which had an area coverage of 1,650.04 square kilometer $(36.45 \%)$ of the land coverage of the park (Table 4). The wetland had also relative large share of the area of the park, 645.99 square kilometer $(14.27 \%)$ of the area coverage as compared with grassland and savannah, comprising $8.74 \%$ and $1.52 \%$ of the land coverage, respectively.

\section{Conclusion}

It can be concluded that at microlevel the Gambella National Park is classified into 6 vegetation communities, whereas at a large level of the park, it was classified into 5 major habitat types.

\section{Data Availability}

The data used to support the findings of this study are included within the supplementary information file.

\section{Conflicts of Interest}

The authors declare that they have no conflicts of interest.

\section{Acknowledgments}

The authors would like to thank Professor Meinsser and his students, Mrs. Anja Stoetz and Mr. Matthias Fessen of Applied Science Department of Berlin University, for their technical support on image classification of habitat type of 
Gambella National Park, when the authors wanted classified Rapid Eye images for their study area. The authors would like to thank Dr. Ahmed Amdihun and Kassahun Abera for their GIS technical support during the data analysis and Addis Ababa University for funding this research.

\section{References}

[1] EWCO-Ethiopian Wildlife Conservation Organization, Unpublished report, Ethiopian Wildlife Conservation Authority, Addis Abeba, Ethiopia, 1993.

[2] GNPMP 2004, Gambella National Park Management Plan Phase III. Unpubished Document, Gambella People's National Regional State Bureau of Agriculture, SKAPE Consult, Addis Abeba, Ethiopia, 2004.

[3] Gambella Survey, Unpublished Report for Systematic Survey of Gambella National Park, Gambella National Park Aerial Survey Report, Gambella, Ethiopia, 2010.

[4] P. G. Puldeng, D. F. Debela Hunde, and W. Chala, "Benefits gained from woodland resource uses: the cases of lare district, Gambella regional state of Ethiopia. Ethiopia," Journal of Applied Science and Technology, vol. 3, no. 2, pp. 53-60, 2012.

[5] T. Awas, D. Sebsebe, and Tamarat Bekele, "An ecological study of the vegetation of Gambella Region, South western Ethiopia," SINET: Ethiopian Journal of Science, vol. 24, no. 2, pp. 213-228, 2001.

[6] M. Sarell, A. Haney, and C. Tolkamp, Sensitive Ecosystems Inventory: Central Okanagan, 2001-2001. Volume 3 Wildlife Habitat Mapping, Gambella National Park Aerial Survey Report, Gambella, Ethiopia, 2003.

[7] M. Monico and P. Schapira, "Aerial survey, Gambella national park and surrounding areas," Technical report IGAD/BMP, Gambella National Park Aerial Survey Report, Gambella, Ethiopia, 2015.

[8] A. Negussie, Analysis of Land and Vegetation Cover Dynamics Using Remote Sensing \& GIS Techniques Case Study of NechSar National Park, M.Sc. Thesis, Addis Ababa University, Addis Ababa, Ethiopia, 2008.

[9] V. Westhoff and E. van der Maarel, "The Braun-Blaquet approach," in Classification of Plant Communities, R. H. Whittaker, Ed., pp. 287-399, Junk Publishers, The Hague, Netherlands, 1978.

[10] I. Hedberg, I. Friis, and E. Pearson, General part and index, Vol. 8, The National Herbarium, Addis Ababa University, Addis Ababa, Ethiopia, 2009.

[11] S. Edwards, M. Tadess, S. Demissew, and I. Hedberg, Flora of Ethiopia and Eritirea, Vol 2, Part 1, Magnoliaceae to Flacourtiaceae, The National Herbarium, Addis Ababa university, Addis Abeba, Ethiopia, 2000.

[12] S. Edwards and M. Tadess, Flora of Ethiopia and Eritirea, Vol 2, Part 2, Canellaceae to Euphorbiaceae, The National Herbarium, Addis Ababa University, Addis Abeba, Ethiopia, 1995.

[13] I. Hedberg and S. Edwards, Flora of Ethiopia and Eritirea, Vol 3, Pittosporaceae to Araliaceae, The National Herbarium, Addis Ababa University, Addis Abeba, Ethiopia, 1989.

[14] I. Hedberg, S. Edwards, and S. Nemomissa, Flora of Ethiopia and Eritirea, Vol 4, Part 1. Apiaceae to Dipsacaceae, The National Herbarium Addis Ababa University, Addis Ababa, Ethiopia, 2003.

[15] I. Hedberg, Ib Friis, and E. Pearson, Flora of Ethiopia and Eritirea, Vol 1, Lycopodiaceae to Pinaceae, The National Herbarium, Addis Ababa University, Addis Abeba, Ethiopia, 2001.
[16] I. Hedberg, K. Ensermu, S. Edwards, S. Demissew, and E. Pearson, Flora of Ethiopia and Eritirea, Vol 5, Gentianaceae to Lamiaceae, The National Herbarium Addis Ababa University, Addis Ababa, Ethiopia, 2006.

[17] I. Hedberg and S. Edwards, Flora of Ethiopia and Eritirea, Vol 7, Poaceae (Gramineae), The National Herbarium, Addis Ababa University, Addis Abeba, Ethiopia, 1995.

[18] B. McCune and M. J. Mefford, PC-ORD.Multivariate Analysis of Ecological Data Version 4.0, MjM software Design, Gleneden Beach, OR, USA, 1999.

[19] M. E. Biondini and C. D. Bonham, E. F. Redente, Secondary successional patterns in a sagebrush (Artemisia tridentata) community as they relate to soil disturbance and soil biological activity," Vegetatio, vol. 60, no. 1, pp. 25-36, 1985.

[20] B. McCune and J. B. Grace, Analysis of Ecological, MjM software Design, Gleneden Beach, OR, USA, 2002.

[21] P. W. Mieke and K. J. Berry, Permutation Methods:adistance Function Approach. Springer Series in Statistics, SpringerVerlag, New york, NY, USA, 2001.

[22] J. B. Grace, L. Allain, and C. Allen, "Vegetation association in a rate community type-coastal tall grass Prairie," Plant Ecology, vol. 147, no. 1, pp. 105-115, 2000.

[23] M. Redente and P. Legendre, "Species assemblage and indicator species: the need for a flexible asymmetrical approach," Ecological Monographs, vol. 67, no. 3, pp. 345-366, 1997.

[24] G. Gatluak, Y. Kumelachew, and P. Ruediger, Planning for core wildlife area of Gambella national park using habitat classification and mapping, Ph.D. Thesis Submitted to Ethiopian Institute of Architecture Building Construction and City Development, Addis Ababa University, Addis Abeba, Ethiopia, 2017. 